\title{
Liver venous deprivation versus portal vein embolization before major hepatectomy: future liver remnant volumetric and functional changes
}

\author{
Boris Guiu ${ }^{1}$, François Quenet ${ }^{2}$, Fabrizio Panaro ${ }^{3}$, Lauranne Piron ${ }^{1}$, Christophe Cassinotto ${ }^{1}$ \\ Astrid Herrerro $^{3}$, François-Régis Souche ${ }^{3}$, Margaux Hermida ${ }^{1}$, Marie-Ange Pierredon-Foulongne ${ }^{1}$, \\ Ali Belgour ${ }^{1}$, Serge Aho-Glele ${ }^{4}$, Emmanuel Deshayes ${ }^{5}$
}

${ }^{1}$ Department of Radiology, St-Eloi University Hospital, Montpellier, France; ${ }^{2}$ Department of Surgery, Institut du Cancer de Montpellier (ICM), Montpellier, France; ${ }^{3}$ Department of Surgery, St-Eloi University Hospital, Montpellier, France; ${ }^{4}$ Department of Epidemiology, Dijon University Hospital, Dijon, France; ${ }^{5}$ Department of Nuclear Medicine, Institut de Recherche en Cancérologie de Montpellier (IRCM), INSERM U1194, Montpellier, France

Contributions: (I) Conception and design: B Guiu, E Deshayes; (II) Administrative support: B Guiu, S Aho-Glele; (III) Provision of study materials or patients: All authors; (IV) Collection and assembly of data: B Guiu, S Aho-Glele, E Deshayes; (V) Data analysis and interpretation: B Guiu, F Quenet, F Panaro, S Aho-Glele, E Deshayes; (VI) Manuscript Writing: All authors; (VII) Final approval of manuscript: All authors.

Correspondence to: Prof. Boris Guiu, MD, PhD. Department of Radiology (Head), St-Eloi University Hospital, 80 avenue Augustin Fliche, 34295 Montpellier, France. Email: B-guiu@chu-montpellier.fr.

Background: We previously showed that embolization of portal inflow and hepatic vein (HV) outflow (liver venous deprivation, LVD) promotes future liver remnant (FLR) volume (FLR-V) and function (FLR-F) gain. Here, we compared FLR-V and FLR-F changes after portal vein embolization (PVE) and LVD.

Methods: This study included all patients referred for liver preparation before major hepatectomy over 26 months. Exclusion criteria were: unavailable baseline/follow-up imaging, cirrhosis, Klatskin tumor, twostage hepatectomy. 99m Tc-mebrofenin SPECT-CT was performed at baseline and at day 7, 14 and 21 after PVE or LVD. FLR-V and FLR-F variations were compared using multivariate generalized linear mixed models (joint modelling) with/without missing data imputation.

Results: Baseline FLR-F was lower in the LVD $(n=29)$ than PVE group $(n=22)(P<0.001)$. Technical success was $100 \%$ in both groups without any major complication. Changes in FLR-V at day 14 and 21 $(+14.2 \%$ vs. $+50 \%, \mathrm{P}=0.002$; and $+18.6 \%$ vs. $+52.6 \%, \mathrm{P}=0.001)$, and in FLR-F at day 7,14 and $21(+23.1 \%$ vs. $+54.3 \%, \mathrm{P}=0.02 ;+17.6 \%$ vs. $+56.1 \%, \mathrm{P}=0.006$; and $+29.8 \%$ vs. $+63.9 \%, \mathrm{P}<0.001)$ differed between $\mathrm{PVE}$ and LVD group. LVD $(\mathrm{P}=0.009)$, age $(\mathrm{P}=0.027)$ and baseline FLR-V $(\mathrm{P}=0.001)$ independently predicted FLR-V variations, whereas only LVD $(\mathrm{P}=0.01)$ predicted FLR-F changes. After missing data handling, LVD remained an independent predictor of FLR-V and FLR-F variations.

Conclusions: LVD is safe and provides greater FLR-V and FLR-F increase than PVE. These results are now evaluated in the HYPERLIV-01 multicenter randomized trial.

Keywords: Portal vein embolization (PVE); liver venous deprivation (LVD); resection; hepatectomy; mebrofenin

Submitted Dec 02, 2019. Accepted for publication Feb 21, 2020.

doi: $10.21037 / \mathrm{hbsn} .2020 .02 .06$

View this article at: http://dx.doi.org/10.21037/hbsn.2020.02.06 


\section{Introduction}

Liver resection is the only curative treatment for patients with large or multiple liver tumors. Over the past decades, advances in surgical techniques and postoperative management have increased the proportion of patients amenable to curative treatment. However, adequate future liver remnant (FLR) remains the main limiting factor to achieve safe R0/R1 resection $(1,2)$. Indeed, posthepatectomy liver failure (PHLF) is still the main cause of death after resection of three or more liver segments $(1,3)$. It is generally accepted that PHLF can occur when FLR volume (FLR-V) is smaller than $25 \%$ of the total volume of a normal liver, $30 \%$ of a liver with steatosis and in patients who had many chemotherapy cycles, and $40 \%$ of a liver with cholestasis or cirrhosis (3).

By occluding the portal venous branches that supply the tumor-bearing liver to be resected, portal vein embolization (PVE) is the standard of care to increase the FLR and to allow safe resection (4). However, FLR size increase takes time (usually 4-12 weeks), and this can explain the hepatectomy cancellation rate of approximately $20 \%$, mainly due to tumor progression $(1,5,6)$. To overcome this issue, the Associating Liver Partition and Portal vein Ligation for Staged hepatectomy (ALPPS) method was proposed in 2012 (7). By partitioning the liver in addition to portal vein ligation, ALPPS accelerates FLR growth $(80 \%$ increase in 1 week), allowing liver resection in the presence of adequate FLR-V after only 7-10 days (8). However, the first results from the analysis of the international ALPPS registry showed unacceptable morbidity/mortality rates and surprisingly high PHLF incidence (9). Some insights came from studies that evaluated the post-ALPPS liver function using ${ }^{99 \mathrm{~m}} \mathrm{Tc}-\mathrm{mebrofenin}$ hepatobiliary scintigraphy, a quantitative method developed to assess total and regional liver function (10). ${ }^{99 \mathrm{~m}} \mathrm{Tc}-$ mebrofenin is taken up by hepatocytes and directly excreted into the bile canaliculi without undergoing any biotransformation (11). This technique predicts the risk of postoperative PHLF with greater accuracy than any other FLR-V evaluation method $(2,10)$. After ALPPS, FLR function (FLR-F) increase was much lower than FLR-V increase, and even strongly decreased in some patients (11-13). Based on these results, FLR-F has gained considerable interest for monitoring the post-embolization FLR changes.

Recently, we described the liver venous deprivation (LVD) technique (14) that associates concomitant portal vein and hepatic vein (HV) embolization in the liver to be resected. Like PVE, LVD is a full trans-hepatic procedure for occluding the entire or part of the venous outflow (right, accessory right, with/without middle HVs). In the first report (15), we observed promising FLR-V growth $(+52.6 \%$ at day 23). Moreover, using ${ }^{99 \mathrm{~m}} \mathrm{Tc}-$ mebrofenin hepatobiliary scintigraphy in three patients, we detected an important FLR-F increase (+65\%) at day 7 after LVD (16).

A previous study using ${ }^{99 \mathrm{~m}} \mathrm{Tc}-\mathrm{meb}$ rofenin data obtained at week 3-4 after PVE showed that FLR-F increase is more pronounced than that of FLR-V. This suggested that the necessary waiting time until resection could be reduced $(2,17)$. Therefore, early evaluations are required to precisely define the optimal time for liver resection. Unfortunately, data on FLR-V and FLR-F changes over time are lacking. Since 2017, at our institution morphological-functional ${ }^{99 m}$ Tc-mebrofenin SPECT-CT scans are performed each week during the first three weeks after liver preparation.

As our previous work showed that $\operatorname{LVD}(15,16)$ and surgical resection following LVD (18) are safe, we wanted to compare the FLR-V and FLR-F changes over time after LVD and PVE. Therefore, the aim of this study was to use the data collected during the 3 -week ${ }^{99 \mathrm{~m}} \mathrm{Tc}$-mebrofenin SPECT-CT imaging follow-up to compare FLR-V and FLR-F changes after PVE and LVD in patients who will undergo major hepatectomy.

We present the following article in accordance with the STROBE reporting checklist (available at http://dx.doi. org/10.21037/hbsn.2020.02.06).

\section{Methods}

This single center retrospective study was performed in accordance with the Declaration of Helsinki (as revised in 2013). All study participants provided a written informed consent. Our institutional review board approved (authorization number: 2019_CLER-MTP_11-03) the retrospective analysis of their anonymized data.

\section{Study population}

All patients referred to our institution for liver preparation before major hepatectomy between January 2017 and March 2019 were included. Liver preparation was considered after discussion at our weekly multidisciplinary consultation meeting (including at least one interventional radiologist, one oncologist, and one liver surgeon) in the case of small FLR. Small FLR was defined as baseline FLR-V $<30 \%$ of the total liver volume (TLV) (minus the tumor 
volume), or as FRL-F ( ${ }^{99 \mathrm{~m}} \mathrm{Tc}-$ mebrofenin clearance rate) $<2.69 \% / \mathrm{min} / \mathrm{m}^{2}(10)$. Moreover, when the planned resection was considered at risk (I) to be larger than expected, or (II) to cause venous congestion of part of the FLR due to vascular damage or resection, an additional $20 \%$ functional margin was considered (i.e., liver preparation proposed when FLR-F $<3.23 \% / \mathrm{min} / \mathrm{m}^{2}$ ). Exclusion criteria for this study were: lack of baseline ${ }^{99 \mathrm{~m}}$ Tc-mebrofenin SPECT-CT or follow-up data for the first 3 weeks after liver preparation; cirrhosis; Klatskin tumor; and two-stage hepatectomy (due to the difficulty to distinguish between regeneration after the first surgical stage and liver preparation).

\section{Baseline and follow-up imaging}

All patients underwent ${ }^{99 \mathrm{~m}} \mathrm{Tc}$-mebrofenin SPECT-CT imaging using a hybrid scanner (Discovery NMCT570, GE Healthcare, Milwaukee, USA) at baseline (prior to liver preparation) and at day 7,14 and 21 after PVE or LVD.

After injection of $150 \mathrm{MBq}$ of ${ }^{99 \mathrm{~m}} \mathrm{Tc}-\mathrm{meb}$ rofenin (Cholediam, Mediam Pharma, Loos, France), a 6-minute dynamic acquisition was performed to assess the total liver clearance rate (in $\% / \mathrm{min} / \mathrm{m}^{2}$ ) normalized to the body surface area. A fast SPECT acquisition was then performed as described elsewhere (16). Finally, CT images $(2.5 \mathrm{~mm}$ slice thickness) were acquired at the portal venous phase using the same system. The Volumetrix ${ }^{\circledR}$ software (GE Healthcare, Milwaukee, USA) was used to reconstruct SPECT data using an iterative algorithm to produce attenuation corrected images. Co-registration between CT and SPECT images was visually and manually checked and corrected when required. On each CT image, the resection margin was jointly planned by the liver surgeon and the nuclear medicine physician. FLR and TLV were automatically calculated by the workstation (OsiriX MD, Pixmeo, Bernex, Switzerland). Tumor volumes were also segmented and subtracted from the TLV and/or FLR, depending on the tumor(s) location(s). These volumes of interest (VOI) created on CT images were exported to the SPECT attenuation corrected images. The actual ${ }^{99 \mathrm{~m}} \mathrm{Tc}-$ mebrofenin counts in the VOI of FLR and TLV were calculated and the corresponding regional functions were defined as [(total counts in the region of interest VOI/total counts in total liver VOI) $\times$ total liver clearance rate] and expressed as $\% / \mathrm{min} / \mathrm{m}^{2}$.

FLR-V and FLR-F variations from baseline were expressed as percentage of the baseline values.

\section{Liver preparation technique}

All PVE and LVD were performed under general anesthesia by the same interventional radiologist (BG) with 12 years of experience in liver procedures. The choice of technique was left to the interventional radiologist who tended to propose LVD in case of low baseline FLR function. All patients received intravenous prophylactic antibiotics (amoxicillin and clavulanic acid). PVE was performed through right portal access, using n-butyl-cyanoacrylate (Glubran II, GEM, Italy) and ethiodized oil (Lipiodol Ultra-fluide, Guerbet, Aulnay-sous-bois, France) at a 1:7 ratio. Embolization was not extended to segment IV. For LVD procedures, embolization of the right, accessory right (when present), +/- middle HVs was performed percutaneously using oversized Amplatzer Vascular Plugs II (St-Jude Medical, Plymouth, MN) and a mixture of n-butylcyanoacrylate (Purefill, Peters Surgical, Bobigny, France) and ethiodized oil at a 1:2 ratio, as described previously (12). Pain was managed with paracetamol and/or morphine titration when necessary, according to the validated algorithm of our institution. Multivitamin and phosphorus supplementation was administered to all patients, as described elsewhere (12).

Abdominal CT imaging at the portal venous phase was performed at day 1 post-intervention. Technical success was defined as complete embolization of the portal and hepatic vessels.

\section{Liver resection and post-operative follow-up}

Liver resection was performed when FRL-F was above the safe threshold (i.e., $2.69 \% / \mathrm{min} / \mathrm{m}^{2}$ or $3.23 \% / \mathrm{min} / \mathrm{m}^{2}$, depending on the FLR venous outflow and/or in the case of expected large resection). Liver function tests were performed the day before and each day after resection until the patient's discharge. PHLF occurrence was assessed according to the "50-50" criteria (19). Grade IIIA-V postoperative complications according to the DindoClavien classification (20) and mortality at day 90 postsurgery were recorded.

\section{Pathological assessment}

Surgical specimens were entirely evaluated according to our institutional protocols. Tissues were fixed in formalin (10\%). Paraffin tissue sections were stained with hematoxylin and 


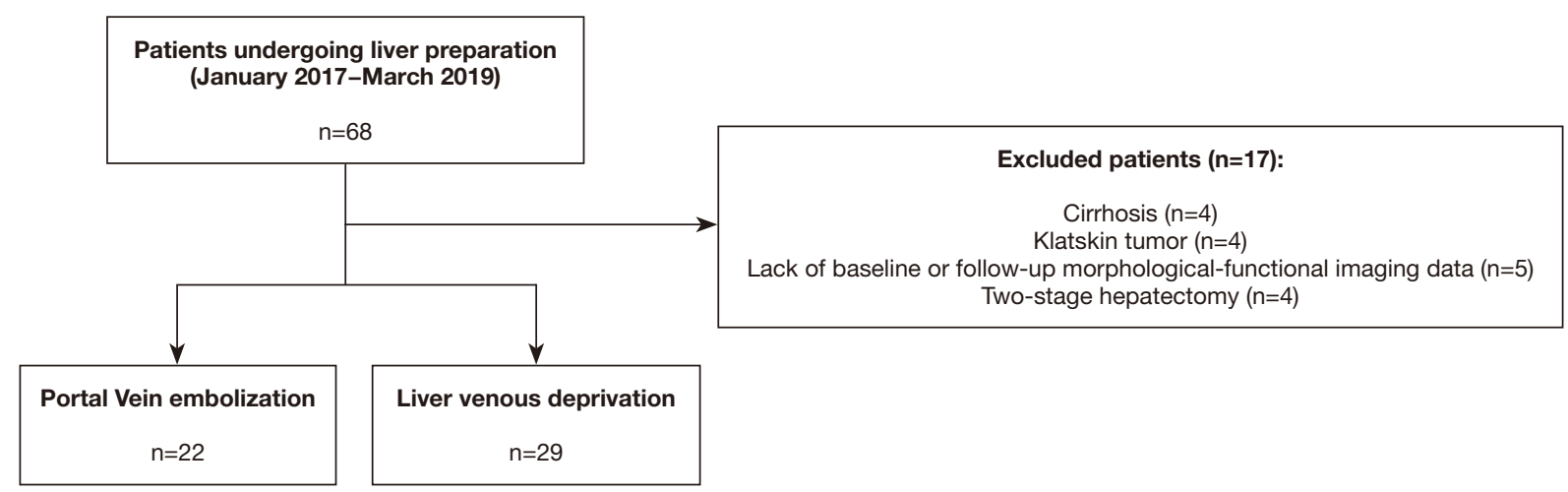

Figure 1 Study flowchart.

eosin or hematoxylin eosin and Safran. Liver samples of the embolized (and resected) lobe were evaluated as described elsewhere (18).

\section{Statistical analyses}

Qualitative and continuous variables were described using numbers, percentages, medians with interquartile ranges (IQR) or range, or means \pm standard deviations (SD).

FLR-V and FLR-F were considered repeated measures because evaluated in each patient at day $0,7,14$ and 21 . FLR-V and FLR-F variations compared with baseline were considered as the outcome variables. To evaluate correlations of repeated measures and the relationship between outcome variables, joint modeling of multiple longitudinal outcomes was performed using multivariate generalized linear mixed models (21). Models were joined on the basis of a shared random effect. A Gaussian distribution of the outcome variables was assumed. Timetrend linearity was checked using the restricted cubic spline regression method.

For each variable, the number of missing data was estimated according to the STROBE guidelines (22). For each outcome variable, a binary variable "missing data, yes or no" was created. The association of these two binary variables with different covariates (such as age, sex...) was studied through a logistic regression model with a clusterrobust variance-covariance estimator (23) to determine the patterns of missingness (e.g., missing at random). First, joint modeling was performed using the available data. Then, missing data were handled using multiple imputation analysis (multivariate normal imputation) (24).

All statistical analyses were performed using the STATA software version 15.0 (Statacorp, College Station, TX, USA); $\mathrm{P}$ values $<0.05$ were considered significant.

\section{Results}

\section{Patients' characteristics (Figure 1 \& Table 1)}

Among the 68 patients who underwent liver preparation during the study period, 17 were excluded because of cirrhosis $(n=4)$, Klatskin tumor $(n=4)$, unavailable baseline/ follow-up morphological-functional imaging $(\mathrm{n}=5)$, or two-stage hepatectomy $(\mathrm{n}=4)$ (Figure 1). Therefore, 51 patients ( 37 men, 14 women) with a median age of 63 years (range, 26-79 years) and median body mass index (BMI) of $26.3 \mathrm{~kg} / \mathrm{m}^{2}$ (range, $16-35.2 \mathrm{~kg} / \mathrm{m}^{2}$ ) were enrolled (Table 1). Among them, 22 underwent PVE and 29 had LVD before resection of liver metastases $(n=17$ and $n=22$, respectively), intra-hepatic cholangiocarcinoma (ICC) $(\mathrm{n}=3$ and $\mathrm{n}=4)$, hepatocellular carcinoma $(\mathrm{n}=1$ and $\mathrm{n}=2)$, epithelioid hemangioendothelioma $(\mathrm{n}=1)$, or liver adenoma $(n=1)$. All patients with metastases $(n=39)$ as well as $3 / 7$ patients with ICC received a median of two cycles (1-4) of systemic chemotherapy for at least 12 weeks. In all patients, chemotherapy was stopped at least 3 weeks before and 3 weeks after liver preparation. The planned surgical procedure was right hemi-hepatectomy $(n=23)$, right hemi-hepatectomy extended to segment IV or I $(n=26)$, or right hemi-hepatectomy extended to segment $\mathrm{IV}+\mathrm{I}(\mathrm{n}=2)$. Baseline \%FLR and FLR-F were 23.4\% (range, 13.6$46.7 \%$ ) and $2.13 \% / \mathrm{min} / \mathrm{m}^{2}$ (range, $1.26-3.13 \% / \mathrm{min} / \mathrm{m}^{2}$ ), respectively. As baseline \%FLR and FLR-F were weakly correlated (Spearman's rho $=0.44, \mathrm{P}=0.001$ ) (Figure 2), they were both investigated. The baseline patients' characteristics 
Table 1 Baseline characteristics of patients prepared by portal vein embolization $(\mathrm{n}=22)$ and liver venous deprivation $(\mathrm{n}=29)$

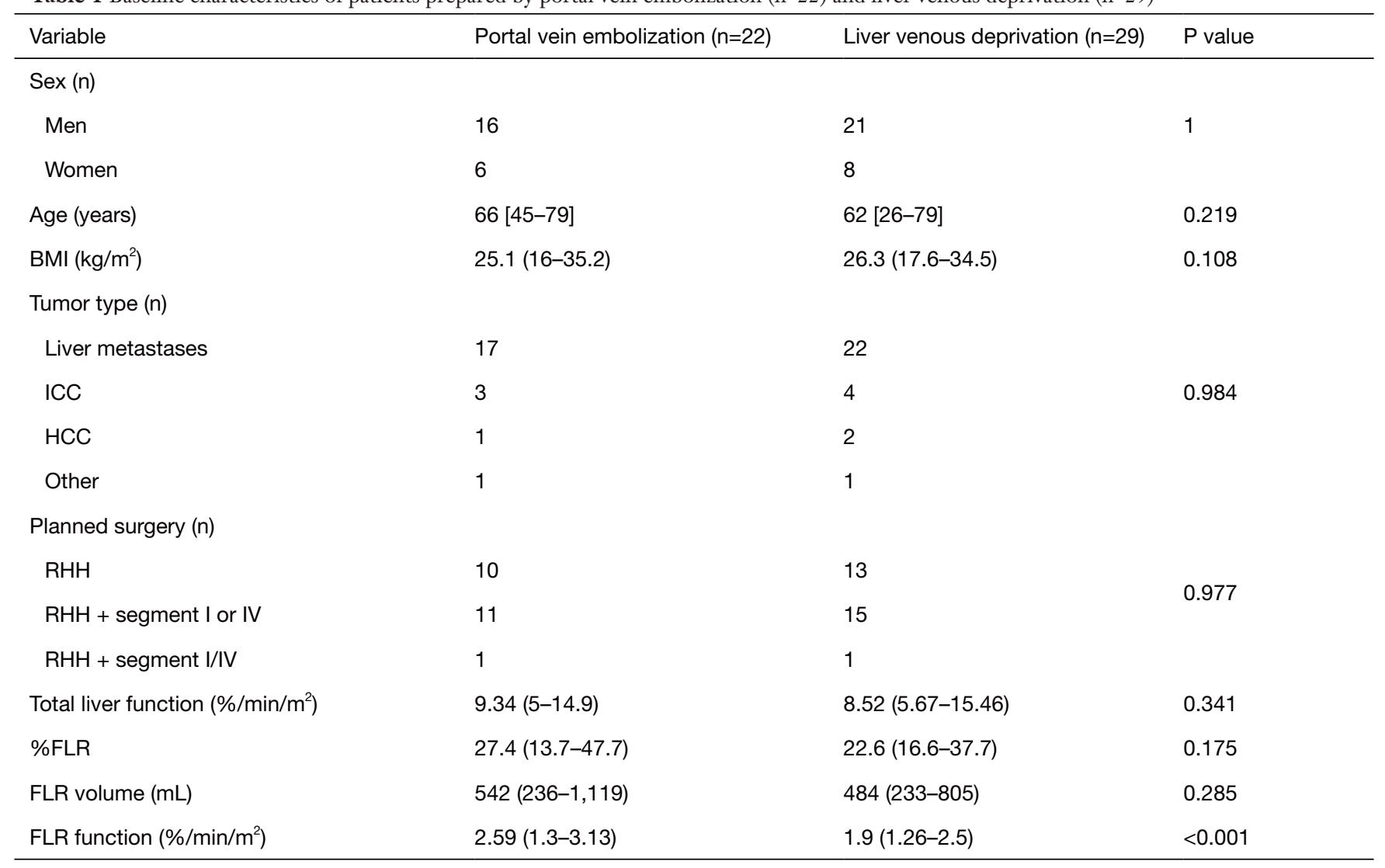

BMI, body mass index; FLR, future liver remnant; ICC, intra-hepatic cholangiocarcinoma; HCC, hepatocellular carcinoma; RHH, right hemi-hepatectomy.

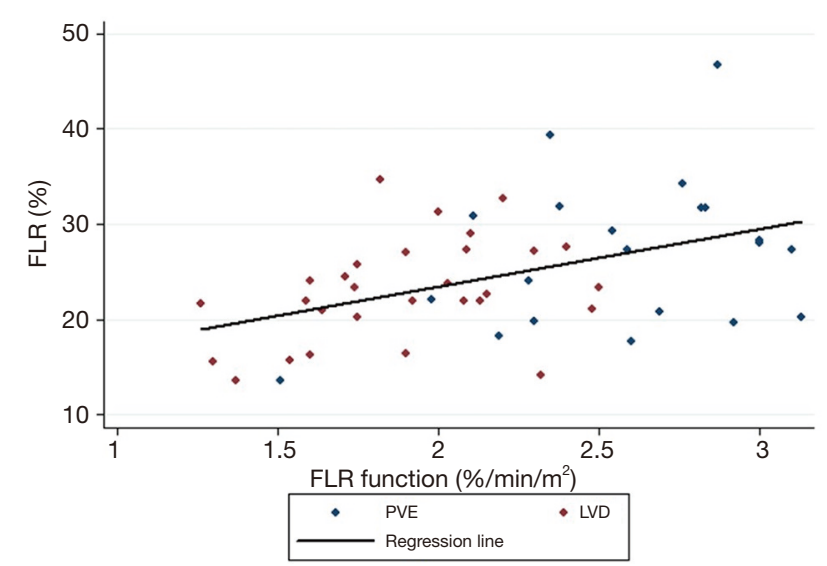

Figure 2 Correlation between baseline \%FLR and FLR function in the PVE (in blue) and LVD (in red) groups (Spearman's rho $=0.44, \mathrm{P}=0.001)$. FLR, future liver remnant; LVD, liver venous deprivation; PVE, portal vein embolization. did not differ between groups (PVE $v s$. LVD), except for baseline FLR-F that was lower in the LVD $\left(1.9 \% / \mathrm{min} / \mathrm{m}^{2}\right)$ than PVE group $\left(2.59 \% / \mathrm{min} / \mathrm{m}^{2}\right)(\mathrm{P}<0.001)$ (Table 1).

\section{Technical LVD and PVE success and tolerance}

Technical success was $100 \%$ in both groups (i.e., embolization of all right portal branches without non-target embolization, and plugs adequately positioned, even in the three patients with accessory right HV). No migration of embolic material was observed. No complication was observed except for minor peri-hepatic hematoma $(n=1$ in the PVE group, $n=2$ in the LVD group) that did not require any additional procedure or medication. Transient grade 1-2 asthenia was reported by six patients $(n=2$ in the PVE group, $\mathrm{n}=4$ in the LVD group). The median hospital stay was 2 days (range, 2-5 days). 
Table 2 Volume and function variations compared with baseline in patients prepared by portal vein embolization ( $\mathrm{n}=22)$ and liver venous deprivation $(\mathrm{n}=29)$

\begin{tabular}{|c|c|c|c|}
\hline Item & Portal vein embolization, median (min to max) & Liver venous deprivation, median ( $\min$ to max) & $P$ value \\
\hline Day 0-day 7 & 28.7 (-20.4 to 90.2$)$ & $37.8(-4.1$ to 88.3$)$ & 0.23 \\
\hline Day 0-day 14 & $14.2(-23.5$ to 58.6$)$ & $50(-4.4$ to 90.6$)$ & 0.002 \\
\hline Day 0-day 21 & $18.6(-10.7$ to 102.2$)$ & 52.6 (1 to 175.6$)$ & 0.001 \\
\hline Day 0-day 7 & $23.1(-16$ to 86.4$)$ & $54.3(-2$ to 105.6$)$ & 0.02 \\
\hline Day 0-day 14 & $17.6(-20$ to 68.9$)$ & $56.1(-5.2$ to 94.7$)$ & 0.006 \\
\hline Day 0-day 21 & 29.8 (1.1 to 63.9$)$ & $68.2(25.4$ to 121.4$)$ & $<0.001$ \\
\hline \multicolumn{4}{|l|}{ Total liver volume } \\
\hline Day 0-day 21 & $-1.5(-16.3$ to 36$)$ & $12.9(-12.7$ to 66$)$ & 0.03 \\
\hline \multicolumn{4}{|c|}{ Total liver function } \\
\hline Day 0-day 7 & $-3.7(-30.8$ to 49.8$)$ & $-12.2(-35.7$ to 15.2$)$ & 0.08 \\
\hline Day 0-day 14 & $0.1(-41$ to 35$)$ & $-14(-32.8$ to 13.9$)$ & 0.15 \\
\hline Day 0-day 21 & $-9.4(-24.8$ to 11.2$)$ & $-15(-33.5$ to 36.5$)$ & 0.65 \\
\hline
\end{tabular}

FLR, future liver remnant.

\section{Volume and functional analysis (Table 2)}

In total, 174 morphological-functional evaluations were performed, and $40(23.3 \%)$ were missing: $12(23.5 \%)$ at day $7,15(29.4 \%)$ at day 14 , and $13(25.5 \%)$ at day 21 . The number of missing evaluations did not differ between groups $(\mathrm{P}=0.62)$. Figure 3 shows an example of ${ }^{99 \mathrm{~m}} \mathrm{Tc}-$ mebrofenin SPECT-CT follow-up in a patient from the LVD group.

\section{TLV}

Compared with baseline, TLV in the PVE group increased by $15 \%$ and $1.2 \%$ at day 7 and day 14 , and decreased by $1.5 \%$ at day 21 . In the LVD group, it increased by $11.1 \%$, $1.2 \%$, and $12.9 \%$ at day 7,14 and 21 , respectively. Only at day 21 , the TLV variation was significantly different between groups $(\mathrm{P}=0.03)$.

\section{Total liver function}

Compared with baseline, total liver function in the PVE group decreased by $3.7 \%$ at day 7 , and then increased by $0.1 \%$ and $9.4 \%$ at day 14 and 21 . In the LVD group, total liver function first decreased by $12.2 \%$ at day 7 , then increased by $0.1 \%$ at day 14 , and then decreased again by $15 \%$ at day 21 (no significant difference between groups at all time points).

\section{FLR volume (Figure 4)}

Compared with baseline, FLR-V increased by $28.7 \%$ (range, $-20.4 \%$ to $90.2 \%$ ) in the PVE group and by $37.8 \%$ (range, $-4.12 \%$ to $88.3 \%)$ in the LVD group $(\mathrm{P}=0.23)$ at day 7 , by $14.2 \%$ (range, $-23.5 \%$ to $58.6 \%$ ) and $50 \%$ (range, $-4.4 \%$ to $90.6 \%)(\mathrm{P}=0.002)$ at day 14 , and by $18.6 \%$ (range, $-10.7 \%$ to $102.2 \%$ ) and $52.6 \%$ (range, $1-175.6 \%$ ) at day 21 $(\mathrm{P}=0.001)$.

\section{FLR function (Figure 5)}

Compared with baseline, FLR-F increased by $23.1 \%$ (range, $-16 \%$ to $86.4 \%$ ) in the PVE group and by $54.3 \%$ (range, $-2 \%$ to $105.6 \%)$ in the LVD group $(\mathrm{P}=0.02)$ at day 7 , by $17.6 \%$ (range, $-20 \%$ to $68.9 \%$ ) and $56.1 \%$ (range, $-5.2 \%$ to $94.7 \%)(\mathrm{P}=0.006)$ at day 14 , and by $29.8 \%$ (range, $1.1-63.9 \%)$ and $68.2 \%$ (range, $25.4-121.4 \%)(\mathrm{P}<0.001)$ at day 21. 


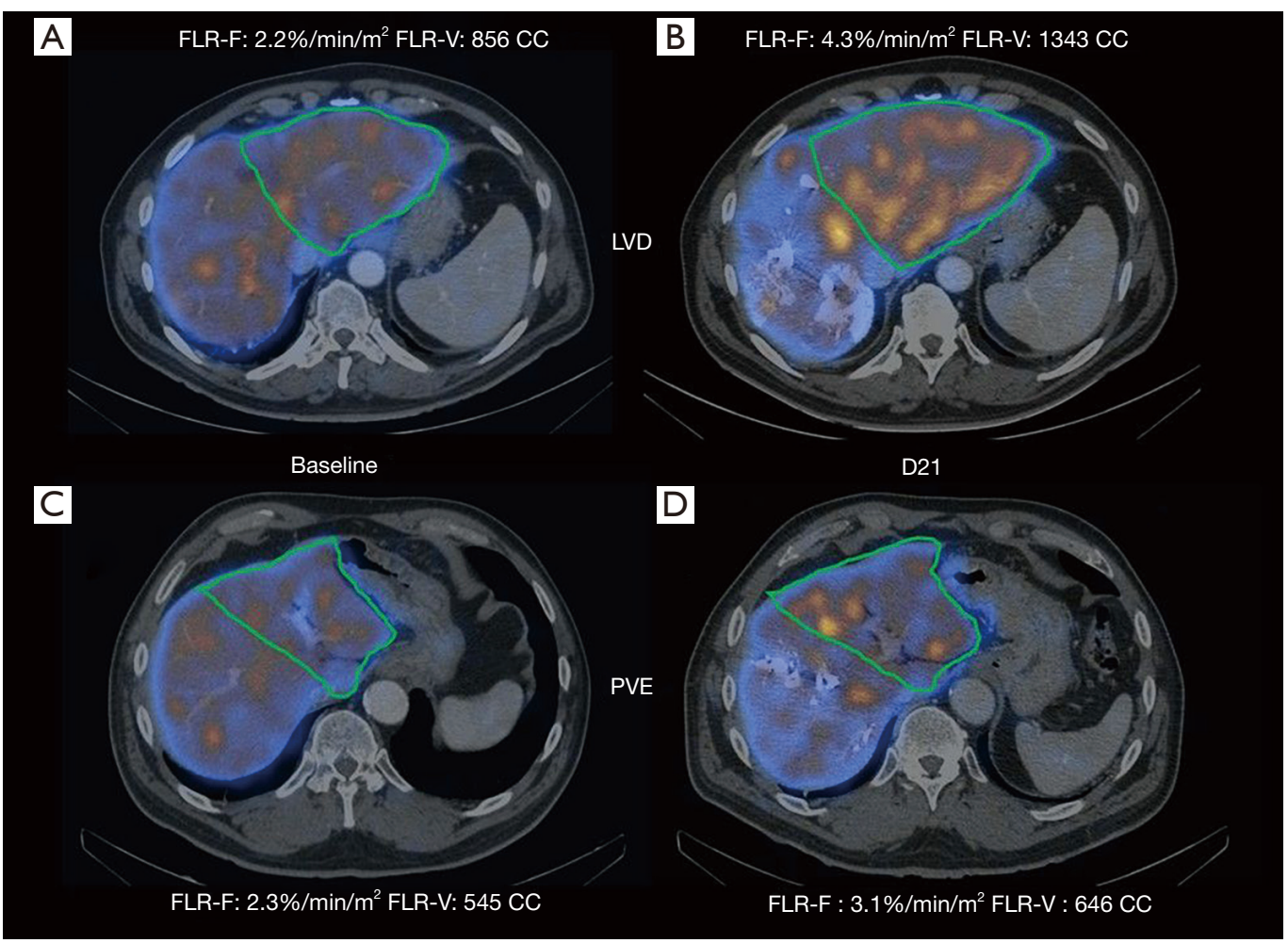

Figure 3 Axial view of 99mTc-mebrofenin SPECT/CT fused images of a patient from the PVE group (upper) and from the LVD group (lower) at baseline (A,C) and at day 21 (B,D) after liver preparation. Green solid lines represent the FLR. FLR, future liver remnant; LVD, liver venous deprivation; PVE, portal vein embolization.

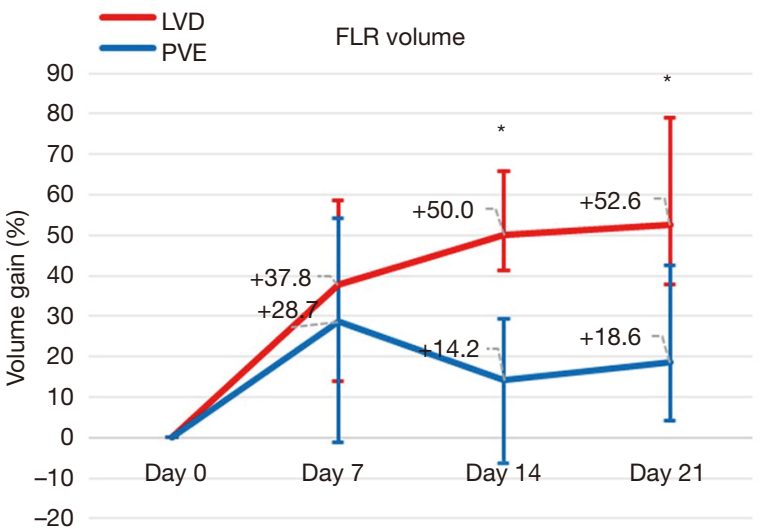

Figure 4 FLR volume increase (median with IQR) from baseline in the PVE (blue) and LVD (red) groups; * indicates statistical significance. FLR, future liver remnant; LVD, liver venous deprivation; PVE, portal vein embolization.

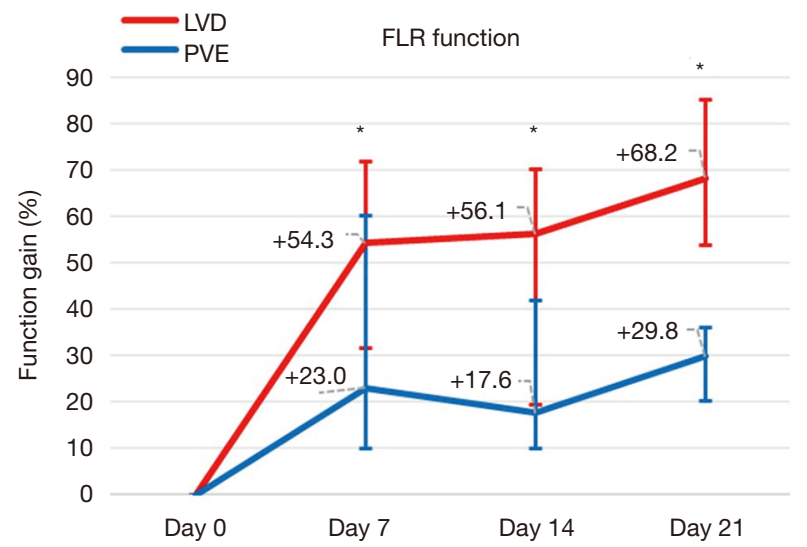

Figure 5 FLR function increase (medians with IQR) from baseline in the PVE (blue) and LVD (red) groups; * indicates statistical significance. FLR, future liver remnant; LVD, liver venous deprivation; PVE, portal vein embolization. 
Table 3 Multivariate analysis (linear mixed models with joint modeling) to identify variables that predict the variation of FLR volume and function (all available data)

\begin{tabular}{lccc}
\hline Covariates & Coefficient & $95 \% \mathrm{Cl}$ & $\mathrm{P}$ value \\
\hline Variation of FLR volume & & & \\
Technique (LVD vs. PVE) & 12.38 & $3.16-21.6$ & 0.009 \\
Age & -0.49 & -0.86 & 0.027 \\
Baseline FLR volume & -0.04 & -0.04 & 0.001 \\
Variation of FLR function & & & \\
Technique (LVD vs. PVE) & 13.55 & $3.23-23.88$ & 0.01 \\
Age & 0.05 & -0.496 & 0.796 \\
Baseline FLR function & -8.82 & -8.288 & 0.066 \\
\hline
\end{tabular}

FLR, future liver remnant; LVD, liver venous deprivation; PVE, portal vein embolization.

Table 4 Multivariate analysis (linear mixed models with joint modeling) to identify variables that predict the variation of FLR volume and function (with missing data imputation)

\begin{tabular}{lccc}
\hline Covariates & Coefficient & $95 \% \mathrm{Cl}$ & $\mathrm{P}$ value \\
\hline Variation of FLR volume & & & \\
Technique (LVD vs. PVE) & 13.9 & 2.31 to 25.49 & 0.021 \\
Age & -0.5 & -1.19 & 0.09 \\
Baseline FLR volume & -0.04 & -0.047 & 0.003 \\
Variation of FLR function & & & \\
Technique (LVD vs. PVE) & 14.66 & 3.23 to 23.88 & 0.021 \\
Age & 0.08 & -0.6 & 0.732 \\
Baseline FLR function & -7.31 & -18.43 to 3.8 & 0.193 \\
\hline
\end{tabular}

FLR, future liver remnant; LVD, liver venous deprivation; PVE, portal vein embolization.

\section{Predictive factors of FLR-V and FLR-F variations}

Liver preparation technique $(\mathrm{P}<0.001)$, age $(\mathrm{P}=0.001)$ and baseline FLR-V $(\mathrm{P}<0.001)$ were associated with FRL-V variations in univariate analysis (linear generalized mixed model), but not TLV, tumor type, and surgery type. Only liver preparation technique $(\mathrm{P}<0.001)$ and baseline FLR-F $(\mathrm{P}<0.001)$ were associated with $\mathrm{FRL}-\mathrm{F}$ variations in univariate analysis (linear generalized mixed model).

Based on the available data, multivariate analysis using joint modeling (of both FLR-V and FLR-F) showed that liver preparation technique (coefficient $=12.38, \mathrm{P}=0.009$ ), age (coefficient $=-0.49, \mathrm{P}=0.027$ ) and baseline FLR-V (coefficient $=-0.04, \mathrm{P}=0.001$ ) were independent predictors of FLR-V variation, whereas liver preparation technique (coefficient $=13.55, \mathrm{P}=0.01$ ) was the only predictor of FLR-F variation (Table 3). After missing data imputation, multivariate analysis confirmed that liver preparation technique (coefficient $=13.9, \mathrm{P}=0.021$ ) and baseline FLR-V (coefficient $=-0.04, \mathrm{P}=0.003$ ) were independent predictors of FLR-V variation, and liver preparation technique (coefficient $=14.66, \mathrm{P}=0.023$ ) was the only predictor of FLR-F variation (Table 4).

\section{Liver surgery}

At week 3 post-preparation, five patients did not reach the safe FLR-F threshold ( $n=2$ in the LVD and $n=3$ in the PVE group). Two of the patients in the PVE group had baseline FLR-F $>1.72 \% / \mathrm{min} / \mathrm{m}^{2}$, which has been proposed as the threshold to identify patients who cannot reach the safe FLR-F cut-off after PVE (25). Two of these five patients ( $n=1$ /group) reached the adequate FLR-F threshold at week 5 post-preparation and underwent surgery, whereas the other three received only systemic treatment. In total, tumor resection was performed in 47 patients (in another one resection was canceled due to peritoneal carcinomatosis discovered at surgery) after a median interval of 32 days (range, 22-46 days) in the LVD and 36 days (range, 2255 days $)$ in the PVE group $(\mathrm{P}=0.12)$. No PHLF was reported according to the "50-50" criteria. At post-operative day 5 , median serum bilirubin concentration was $34 \mu \mathrm{mol} / \mathrm{L}$ (IQR, 17-43 $\mu \mathrm{mol} / \mathrm{L}$ ) in LVD and $38 \mu \mathrm{mol} / \mathrm{L}$ (IQR, $18-44 \mu \mathrm{mol} / \mathrm{L})$ in PVE patients $(\mathrm{P}=0.38)$ whereas corresponding PT values were 67\% (IQR, 56-86\%) and $65 \%$ (IQR, 54-80\%) ( $\mathrm{P}=0.56)$, respectively. Major complications (Clavien-Dindo $\geq$ IIIA) occurred in three patients in each group. One patient from the PVE group died from pulmonary embolism.

\section{Histological assessment}

Eighteen $(66.7 \%)$ and nine $(45 \%)$ patients presented important sinusoidal dilatation respectively after PVE and LVD $(\mathrm{P}=0.21)$. Centro- and medio-lobular hepatic plates exhibited major atrophy (especially in the vicinity of sinusoidal dilatation) in 18 (66.7\%) patients after LVD and in $8(40 \%)$ patients after PVE $(\mathrm{P}=0.07)$. Hemorrhage and necrosis were observed in $13(48.1 \%)$ patients after LVD and in $7(35 \%)$ patients after $\mathrm{PVE}(\mathrm{P}=0.37)$. 


\section{Discussion}

To our knowledge, this is the first study to compare early FLR-V and FLR-F changes following PVE and LVD. We recently described the LVD technique that combines the concomitant embolization of portal inflow and venous outflow $(14,16)$. Occluding the hepatic venous outflow, in addition to PVE, may improve FLR regeneration through several mechanisms. Hepatic venous embolization (HVE) can counteract persistent minute portal inflow that may occur after incomplete or too proximal PVE by reversing the portal inflow. In addition, porto-portal collateralization between the liver portions with and without portal embolization should be strongly reduced due to outflow obstruction, as recently shown in a preclinical pig model (26). Moreover, occlusion of the hepatic venous outflow in a PVE context decreases the hepatic arterial inflow due to the hepatic arterial buffer response (27), and therefore can increase damage to the liver portion that underwent embolization (15). In the absence of any significant difference in histological damage of the embolized liver, we can speculate that counteraction of persistent minute portal inflow and reduction in porto-portal collateralization are the predominant mechanisms explaining the effects of LVD.

In this study, we confirmed that LVD is as safe as PVE. In the initial experience, we reported deep asthenia in some patients (16). However, after the introduction of post-LVD vitamin and ion supplementation, we did not observe this complication any longer, confirming that LVD is an energydemanding technique. In another study, we showed that the perioperative impact of LVD and PVE was comparable in patients undergoing right hemi-hepatectomy (18). The absence of PHLF in our series contrasts with the commonly reported rates $(2-16 \%)$ after resection of colorectal metastases $(28,29)$, where up to $7 \%$ of severe PHLF was observed despite adequate FLR-V. The decision to operate only when FLR-F was above the safe threshold probably explains the absence of PHLF, in agreement with previous studies $(2,30)$.

Given these promising preliminary results $(15,16)$, it was necessary to compare FLR changes after LVD and PVE. During the first three weeks after PVE, we observed a $14.2-28.7 \%$ FLR-V gain, within the range of what is commonly reported at week $2-8$ after PVE $(8-37.9 \%)(1,6)$. However, comparison with previous data is difficult due to the considerable variability in time intervals of FLR growth follow-up by imaging (31).

In our study, baseline FLR-V was independently (and negatively) associated with FLR-V gain, as reported elsewhere (32). This point is of great importance and probably underestimated in the literature. It means that the FLR-V volume gain is higher with small baseline FLR-V.

FLR-V increase after LVD was faster and greater $(+37.8 \%$ at week $1,+52.6 \%$ at week 3$)$, although regeneration seems more important after liver resection [ $\approx 55 \%$ at post-operative day $7(33,34)]$ and ALPPS [+48.6\% to $90 \%$ between stage 1 and $2(7,35,36)]$. However, functional regeneration is delayed compared with volumetric regeneration after liver resection (37). The same applies after ALPPS stage 1 where FLR-F gain, evaluated by ${ }^{99 \mathrm{~m}} \mathrm{Tc}-\mathrm{mebrofenin}$ scintigraphy, is 2-fold lower than FLR-V increase (38-40). Conversely after PVE, FLR-F gain is greater than FLR-V gain $(2,17)$. Therefore, although baseline FLR-V and FLR-F are correlated (as confirmed in our study), the variation of one cannot be used to predict changes in the other.

By capturing both FLR quality and quantity, ${ }^{99 \mathrm{~m}} \mathrm{Tc}-$ mebrofenin morphological-functional imaging brings meaningful data for comparing LVD and PVE effects. Our results show that FLR-F increase is greater and faster after LVD than after PVE. As FLR-F and FLR-V were concomitantly evaluated using the same imaging modality ${ }^{99 \mathrm{~m}} \mathrm{Tc}-$ mebrofenin SPECT/CT), we chose joint modeling to investigate the effect of the preparation technique (LVD vs. PVE) on FLR variation. We adjusted the analysis for (I) age, because liver function declines with age (41), (II) baseline FLR-V because it is inversely correlated with FLR-V gain (32), and (III) baseline FLR-F because it was significantly different in the LVD and PVE groups (we tended to propose LVD, the more aggressive technique, mainly to patients with low baseline FLR-F). Multivariate analysis showed that LVD independently predicted greater FLR-V and FLR-F increase, with/without missing data imputation.

In a very recent retrospective study, greater FLR hypertrophy at week 3-4 was observed after embolization of the portal and hepatic veins than after PVE $(+51.2 \% v s$. $+31.9 \%, \mathrm{P}=0.018)(42)$. Unlike LVD, in HVE one plug is deployed proximally in one HV (usually the right) through the trans-jugular access (43). In LVD, the objective is the proximal occlusion of one/several major $\mathrm{HV}(\mathrm{s})$, and also the embolization of distal venous branches to limit the development of veno-venous collaterals. Such collaterals have been detected in $46 \%$ of normal livers $(44,45)$ and may rapidly enlarge after plug deployment $(15,16)$. Comparable FLR-V gains were reported in this study $(+52.6 \%$ at day 21 after LVD) and that of Le Roy et al. $(+51.2 \%$ at 
day 26 after bi-embolization) (42). As both assessment time [day 21 (LVD) vs. 26 (bi-embolization)] and baseline FLR-V [394 cc (bi-embolization) (42) vs. 484 cc (LVD); +22.8\%] influence FLR-V gain, we can hypothesize that volume gain is actually greater after LVD than after bi-embolization, although this would require another dedicated study.

One of the most striking result of our study is the very high FLR-F gain after LVD (+68.2\% at week 3$)$, unprecedented for a liver preparation technique. Although very few studies have investigated FLR-F after PVE $(2,17)$, FLR-F increase by 51.9\% was reported at week 3-4 after PVE. Here, we observed an increase by $54.3 \%$ at day 7 after LVD. A very recent study on 125 patients undergoing major hepatectomy (34) showed that median FLR-F increase at post-operative day 7 was $38.8 \%$. Therefore LVD can provide a greater FLR-F gain than hepatectomy does itself.

In the ALPPS literature (11-13), despite extremely rapid FLR hypertrophy, FLR-F was increased by only $22-28 \%$ at day $6-7$, a value comparable to the one we obtained after PVE. LVD provides at least a 2-fold greater increase at the same time point. In addition, Olthof et al. reported a surprising FLR-F decrease in 6/27 patients after ALPPS stage 1 (11). In another series, Sparrelid et al. (12) showed that FLR-F could even decrease by $-35.7 \%$. This unpredictable FLR-F degradation after ALPPS stage 1 should be a severe warning against this technique, and may explain the high PHLF rate (14\%) reported in the ALPPS registry (9). Conversely, only one of our 29 patients who underwent LVD presented a very limited and transient FLR-F decrease ( $-2 \%$ at day 7 and $-5 \%$ at day 14$)$ that was reversed at day $21(+25.4 \%)$.

Several limitations must be acknowledged. Fist, the number of included patients was relatively small. However, they were consecutively enrolled and PVE, which is part of both procedures, was performed by the same operator using exactly the same technique. Second, although all the other baseline characteristics were comparable, baseline FLR-F was lower in the LVD group because we tended to propose the more aggressive technique to these patients. Therefore, we adjusted the analysis for this covariate to limit the selection bias. Third, some data are missing as generally observed in retrospective studies. Some morphologicalfunctional evaluations were not performed for several reasons, such as patient's opposition, ${ }^{99 \mathrm{~m}} \mathrm{Tc}-\mathrm{meb}$ rofenin shortage, or earlier achievement of the safe FLR-F threshold. Nevertheless, the analyses performed before and after missing data imputation gave the same results. Fourth, although joint modeling, adjustment for covariates and missing data imputation were performed, the higher FLR increase after LVD must be confirmed in a prospective multicenter trial. To this aim, the French multicenter randomized phase II HYPERLIV-01 (NCT03841305) trial is currently recruiting patients.

This retrospective study confirms that the statistical hypothesis on the primary endpoint of the HYPERLIV-01 trial ( $\geq 12 \%$ of difference in FRL- $V$ at week 3 between LVD and PVE) is realistic. Finally, faster and greater liver regeneration after LVD could both increase the proportion of patients amenable to liver resection and reduce the waiting time from preparation to resection, limiting surgery cancellation due to tumor progression. However, fast regeneration could also favor tumor progression and ultimately alter the oncologic outcome. This critical point could not be properly evaluated in our study due to the variability in tumor types. In the HYPERLIV-01 trial, only patients with colorectal cancer liver metastases are enrolled to allow the reliable assessment of the oncological outcomes.

\section{Conclusions}

In conclusion, LVD is safe and provides greater FLR-V and particularly FLR-F, compared with PVE. These results need to be confirmed in the ongoing HYPERLIV-01 multicenter randomized trial.

\section{Acknowledgments}

The authors thank Elisabetta Andermarcher for revising the English.

Funding: None.

\section{Footnote}

Reporting Checklist: The authors have completed the STROBE reporting checklist. Available at http://dx.doi. org/10.21037/hbsn.2020.02.06

Data Sharing Statement: Available at http://dx.doi. org/10.21037/hbsn.2020.02.06

Conflicts of Interest: All authors have completed the ICMJE uniform disclosure form (available at http://dx.doi. org/10.21037/hbsn.2020.02.06). The authors have no conflicts of interest to declare. 
Ethical Statement: The authors are accountable for all aspects of the work in ensuring that questions related to the accuracy or integrity of any part of the work are appropriately investigated and resolved. This single center retrospective study was performed in accordance with the Declaration of Helsinki (as revised in 2013). All study participants provided a written informed consent. Our institutional review board approved (authorization number: 2019_CLER-MTP_11-03) the retrospective analysis of their anonymized data.

Open Access Statement: This is an Open Access article distributed in accordance with the Creative Commons Attribution-NonCommercial-NoDerivs 4.0 International License (CC BY-NC-ND 4.0), which permits the noncommercial replication and distribution of the article with the strict proviso that no changes or edits are made and the original work is properly cited (including links to both the formal publication through the relevant DOI and the license). See: https://creativecommons.org/licenses/by-nc-nd/4.0/.

\section{References}

1. Abulkhir A, Limongelli P, Healey AJ, et al. Preoperative portal vein embolization for major liver resection: a metaanalysis. Ann Surg 2008;247:49-57.

2. de Graaf W, van Lienden KP, van den Esschert JW, et al. Increase in future remnant liver function after preoperative portal vein embolization. Br J Surg 2011;98:825-34.

3. Asencio JM, Vaquero J, Olmedilla L, et al. "Small-forflow" syndrome: shifting the "size" paradigm. Med Hypotheses 2013;80:573-7.

4. Abdalla EK, Barnett CC, Doherty D, et al. Extended hepatectomy in patients with hepatobiliary malignancies with and without preoperative portal vein embolization. Arch Surg 2002;137:675-80; discussion 680-1.

5. Pommier R, Ronot M, Cauchy F, et al. Colorectal liver metastases growth in the embolized and non-embolized liver after portal vein embolization: influence of initial response to induction chemotherapy. Ann Surg Oncol 2014;21:3077-83.

6. van Lienden KP, van den Esschert JW, de Graaf W, et al. Portal vein embolization before liver resection: a systematic review. Cardiovasc Intervent Radiol 2013;36:25-34.

7. Schnitzbauer AA, Lang SA, Goessmann H, et al. Right portal vein ligation combined with in situ splitting induces rapid left lateral liver lobe hypertrophy enabling 2 -staged extended right hepatic resection in small-for-size settings. Ann Surg 2012;255:405-14.

8. Schadde E, Ardiles V, Robles-Campos R, et al. Early survival and safety of ALPPS: first report of the International ALPPS Registry. Ann Surg 2014;260:829-36; discussion 836-8.

9. Schadde E, Raptis DA, Schnitzbauer AA, et al. Prediction of Mortality After ALPPS Stage-1: An Analysis of 320 Patients From the International ALPPS Registry. Ann Surg 2015;262:780-5; discussion 785-6.

10. de Graaf W, van Lienden KP, Dinant S, et al. Assessment of future remnant liver function using hepatobiliary scintigraphy in patients undergoing major liver resection. J Gastrointest Surg 2010;14:369-78.

11. Olthof PB, Tomassini F, Huespe PE, et al. Hepatobiliary scintigraphy to evaluate liver function in associating liver partition and portal vein ligation for staged hepatectomy: Liver volume overestimates liver function. Surgery 2017;162:775-83.

12. Sparrelid E, Jonas E, Tzortzakakis A, et al. Dynamic Evaluation of Liver Volume and Function in Associating Liver Partition and Portal Vein Ligation for Staged Hepatectomy. J Gastrointest Surg 2017;21:967-74.

13. Tomassini F, D'Asseler Y, Giglio MC, et al. Hemodynamic changes in ALPPS influence liver regeneration and function: results from a prospective study. HPB (Oxford) 2019;21:557-65.

14. Guiu B, Chevallier P, Denys A, et al. Simultaneous transhepatic portal and hepatic vein embolization before major hepatectomy: the liver venous deprivation technique. Eur Radiol 2016;26:4259-67.

15. Guiu B, Chevallier P, Denys A, et al. Simultaneous transhepatic portal and hepatic vein embolization before major hepatectomy: the liver venous deprivation technique. Eur Radiol 2016;26:4259-67.

16. Guiu B, Quenet F, Escal L, et al. Extended liver venous deprivation before major hepatectomy induces marked and very rapid increase in future liver remnant function. Eur Radiol 2017;27:3343-52.

17. Rassam F, Olthof PB, van Lienden KP, et al. Functional and volumetric assessment of liver segments after portal vein embolization: Differences in hypertrophy response. Surgery 2019;165:686-95.

18. Panaro F, Giannone F, Riviere B, et al. Perioperative impact of liver venous deprivation compared with portal venous embolization in patients undergoing right hepatectomy: preliminary results from the pioneer center. Hepatobiliary Surg Nutr 2019;8:329-37. 
19. Balzan S, Belghiti J, Farges O, et al. The "50-50 criteria" on postoperative day 5: an accurate predictor of liver failure and death after hepatectomy. Ann Surg 2005;242:824-8, discussion 828-9.

20. Clavien PA, Barkun J, de Oliveira ML, et al. The ClavienDindo classification of surgical complications: five-year experience. Ann Surg 2009;250:187-96.

21. Crowther MJ. Extended multivariate generalised linear and non-linear mixed effects models. 2017. arXiv:1710.02223.

22. von Elm E, Altman DG, Egger M, Pocock SJ, et al. The Strengthening the Reporting of Observational Studies in Epidemiology (STROBE) statement: guidelines for reporting observational studies. Lancet 2007;370:1453-7.

23. Cameron A, Miller D. A Practitioner's Guide to Cluster-Robust Inference. Journal of Human Resources 2015;50:317-72.

24. De Silva AP, Moreno-Betancur M, De Livera AM, et al. A comparison of multiple imputation methods for handling missing values in longitudinal data in the presence of a time-varying covariate with a non-linear association with time: a simulation study. BMC Med Res Methodol 2017;17:114.

25. Cieslak KP, Huisman F, Bais T, et al. Future remnant liver function as predictive factor for the hypertrophy response after portal vein embolization. Surgery 2017;162:37-47.

26. Schadde E, Guiu B, Deal R, et al. Simultaneous hepatic and portal vein ligation induces rapid liver hypertrophy: A study in pigs. Surgery 2019;165:525-33.

27. Ko GY, Hwang S, Sung KB, et al. Interventional oncology: new options for interstitial treatments and intravascular approaches: right hepatic vein embolization after right portal vein embolization for inducing hypertrophy of the future liver remnant. J Hepatobiliary Pancreat Sci 2010;17:410-2.

28. Narita M, Oussoultzoglou E, Bachellier P, et al. Posthepatectomy liver failure in patients with colorectal liver metastases. Surg Today 2015;45:1218-26.

29. Vibert E, Pittau G, Gelli M, et al. Actual incidence and long-term consequences of posthepatectomy liver failure after hepatectomy for colorectal liver metastases. Surgery 2014;155:94-105.

30. Cieslak KP, Bennink RJ, de Graaf W, et al. Measurement of liver function using hepatobiliary scintigraphy improves risk assessment in patients undergoing major liver resection. HPB (Oxford) 2016;18:773-80.

31. Corrêa D, Schwartz L, Jarnagin WR, et al. Kinetics of liver volume changes in the first year after portal vein embolization. Arch Surg 2010;145:351-4; discussion 354-5.

32. de Baere T, Teriitehau C, Deschamps F, et al. Predictive factors for hypertrophy of the future remnant liver after selective portal vein embolization. Ann Surg Oncol 2010;17:2081-9.

33. Zappa M, Dondero F, Sibert A, et al. Liver regeneration at day 7 after right hepatectomy: global and segmental volumetric analysis by using CT. Radiology 2009;252:426-32.

34. Truant S, Baillet C, Fulbert M, et al. Asymmetric kinetics of volume and function of the remnant liver after major hepatectomy as a key for postoperative outcome - A casematched study. HPB (Oxford) 2020;22:855-63.

35. Truant S, Scatton O, Dokmak S, et al. Associating liver partition and portal vein ligation for staged hepatectomy (ALPPS): impact of the inter-stages course on morbimortality and implications for management. Eur J Surg Oncol 2015;41:674-82.

36. Buac S, Schadde E, Schnitzbauer AA, et al. The many faces of ALPPS: surgical indications and techniques among surgeons collaborating in the international registry. $\mathrm{HPB}$ 2016;18:442-8.

37. Kwon AH, Matsui Y, Kaibori M, et al. Functional hepatic regeneration following hepatectomy using galactosylhuman serum albumin liver scintigraphy. Transplant Proc 2004;36:2257-60.

38. Truant S, Baillet C, Deshorgue AC, et al. Drop of Total Liver Function in the Interstages of the New Associating Liver Partition and Portal Vein Ligation for Staged Hepatectomy Technique: Analysis of the "Auxiliary Liver" by HIDA Scintigraphy. Ann Surg 2016;263:e33-4.

39. Oldhafer F, Ringe KI, Timrott K, et al. Monitoring of liver function in a 73-year old patient undergoing 'Associating Liver Partition and Portal vein ligation for Staged hepatectomy': case report applying the novel liver maximum function capacity test. Patient Saf Surg 2016;10:16.

40. Truant S, Baillet C, Deshorgue AC, et al. Hepatobiliary Scintigraphy in ALPPS: A Response. Ann Surg 2017;266:e112-3.

41. Cieslak KP, Baur O, Verheij J, et al. Liver function declines with increased age. HPB (Oxford) 2016;18:691-6.

42. Le Roy B, Gallon A, Cauchy F, et al. Combined biembolization induces higher hypertrophy than portal vein embolization before major liver resection. $\mathrm{HPB}$ 
(Oxford) 2020;22:298-305.

43. Le Roy B, Perrey A, Fontarensky M, et al. Combined Preoperative Portal and Hepatic Vein Embolization (Biembolization) to Improve Liver Regeneration Before Major Liver Resection: A Preliminary Report. World J Surg 2017;41:1848-56.

44. Gai YH, Cai SF, Guo WB, et al. Sonographic

Cite this article as: Guiu B, Quenet F, Panaro F, Piron L, Cassinotto C, Herrerro A, Souche FR, Hermida M, PierredonFoulongne MA, Belgour A, Aho-Glele S, Deshayes E. Liver venous deprivation versus portal vein embolization before major hepatectomy: future liver remnant volumetric and functional changes. HepatoBiliary Surg Nutr 2020;9(5):564-576. doi: 10.21037/hbsn.2020.02.06 classification of draining pathways of obstructed hepatic veins in Budd-Chiari syndrome. J Clin Ultrasound 2014;42:134-42.

45. Hribernik M, Trotovsek B. Intrahepatic venous anastomoses with a focus on the middle hepatic vein anastomoses in normal human livers: anatomical study on liver corrosion casts. Surg Radiol Anat 2014;36:231-7. 